\title{
Imprecise Approaches to Analysis of Insurance Portfolio with Catastrophe Bond
}

\author{
Maciej Romaniuk ${ }^{(\otimes)}(\mathbb{D}$ \\ Systems Research Institute, Polish Academy of Sciences, \\ Newelska 6, 01-447 Warsaw, Poland \\ mroman@ibspan.waw.pl
}

\begin{abstract}
In this paper, imprecise approaches to model the risk reserve process of an insurer's portfolio, which consists of a catastrophe bond and external help, and with a special penalty function in the case of a bankruptcy event, are presented. Apart from the general framework, two special cases, when parameters of the portfolio are described by L-R fuzzy numbers or shadowed sets, are discussed and compared. In a few examples based on the real-life data for these two types of impreciseness, some important characteristics of the portfolio, like the expected value and the probability of the ruin, are estimated, analysed and compared using the Monte Carlo simulations.
\end{abstract}

Keywords: Risk process $\cdot$ Fuzzy numbers $\cdot$ Shadowed sets $\cdot$ Insurance portfolio $\cdot$ Numerical simulations

\section{Introduction}

Due to the global warming effect, changes in land use, and other effects related to humans' activities, number and severity of natural catastrophes (like tsunamis, earthquakes, floods etc.) are still increasing. Rising demand for related compensations is a serious problem for the insurers, so they have to introduce new financial and insurance instruments to compensate for their losses. A catastrophe bond (abbreviated as a cat bond, see, e.g., $[7,11,12,14]$ ) is an example of such an instrument, which transfer risks from an insurance market to financial markets (which is known as "securitization of losses"). But these new instruments require more complex approaches to the classical problem: how to calculate the probability of the insurer's ruin? Simulations (like the Monte Carlo method) can be applied to analyse even very complex insurer's portfolios (see, e.g., $[18,20]$ ), which consist of many different instruments (i.e. layers), like the classical risk process, issued catastrophe bonds, a reinsurance contract, etc. A numerical approach is a very convenient tool also in other areas (see, e.g., $[9,15,16])$.

Moreover, future characteristics of natural catastrophes and other aspects of the insurer's market (e.g., level of possible external help after a serious natural catastrophe) are not known precisely. Therefore, an imprecise setting (related to, e.g., fuzzy numbers or shadowed sets) is a natural approach to overcome problems with such partially unknown parameters (see, e.g., [18,23]).

(C) Springer Nature Switzerland AG 2020

M.-J. Lesot et al. (Eds.): IPMU 2020, CCIS 1239, pp. 3-16, 2020

https://doi.org/10.1007/978-3-030-50153-2_1 
The literature devoted to insurance mathematics, especially to the problem of the ruin probability of the insurer, is abundant (see, e.g., $[2,7,10])$. However, the imprecise approach in this area is still underdeveloped (see, e.g., [5] for a review of the literature). The following paper can be treated as a further development of the ideas presented in [18-20,22]. Then, a generalized form of the classical risk process is considered and additional layers of the insurance portfolio (like a catastrophe bond and an external help) under an assumption about the value of money in time are analysed using the MC simulations. Moreover, the imprecise setting is used to describe some parameters of the considered models.

A contribution of this paper is fourfold. First, a general simulation framework which enables us to use many, not only one, imprecise parameters (with values based, e.g., on the experts' opinions) of the considered models for the insurer's portfolio, is presented. Second, this general framework is discussed in a more detailed way for two practically important types of imprecise values: fuzzy numbers (especially L-R fuzzy numbers) and shadowed sets. Third, a generalization of the classical risk reserve process for the complex insurer's portfolio, which consists of an issued catastrophe bond and a (possible) external (e.g., governmental or from other institution) help, together with an embedded penalty function (applied in the case of a bankruptcy event), is presented. Moreover, this generalization uses the assumption about the value of money in time. Fourth, the two imprecise approaches (i.e. for L-R fuzzy numbers and shadowed sets) are numerically compared in a few examples. In these examples, the Monte Carlo (MC) simulations are used to calculate various characteristics of the portfolio, which are important for the insurer (like the expected value of this portfolio or the probability of the insurer's ruin in finite time).

This paper is organized as follows. In Sect.2, the classical risk process is generalized to take into account the previously mentioned additional layers and the assumption about the value of money in time. In Sect. 3, necessary definitions concerning fuzzy numbers and shadowed sets are recalled. In Sect. 4 , the general approach for imprecise settings of the considered models is presented and then applied for L-R fuzzy numbers and shadowed sets in simulations discussed in Sect. 5. The paper is concluded in Sect. 6 with some final remarks.

\section{Model of Insurance Portfolio}

The classical risk reserve process $R_{t}$ is defined as a model of the financial reserves of an insurer depending on time $t$, i.e.

$$
R_{t}=u+p t-C_{t}^{*}
$$

where $u$ is an initial reserve of the insurer, $p$ is a rate of premiums paid by the insureds per unit time and $C_{t}^{*}$ is a claim process of the form

$$
C_{t}^{*}=\sum_{i=1}^{N_{t}} C_{i}
$$


where $C_{1}, C_{2}, \ldots$ are iid random values of the claims. Usually, these claims have the same values as the losses caused by the natural catastrophes $U_{i}$, i.e. $C_{i}=U_{i}$, so we have an additional process of the losses

$$
U_{t}^{*}=\sum_{i=1}^{N_{t}} U_{i}
$$

In [20], the claims were modelled as a deterministic or random part of the losses, i.e. $C_{i}=\alpha_{\text {claim }} Z_{i} U_{i}$, where $\alpha_{\text {claim }} \in[0,1], Z_{i} \sim U\left[c_{\min }, c_{\max }\right]$, and $Z_{i}, U_{i}$ are mutually independent variables. Then, $\alpha_{\text {claim }}$ describes a deterministic share of the considered insurer in the whole insurance market (for the given region) and $Z_{i}$ models a random part of the claim $C_{i}$ in the loss $U_{i}$. In the following, we assume that $\alpha_{\text {claim }}$ is given in the imprecise way to model share of the insurer in the market in a more real-life way, e.g., when this share is not exactly stated, its level varies depending on a region of a possible natural catastrophe or its source, etc. It can lead to some hedging problems (see, e.g., [19,20] for additional details).

The process of a number of the claims (and the losses) $N_{t} \geq 0$ is usually driven by some Poisson process, e.g., a non-homogeneous Poisson process (NHPP). In this paper, we assume an intensity function for this NHPP of the form

$$
\lambda_{\mathrm{NHPP}}(t)=a+b t+c \sin (2 \pi(t+d))+m \exp \left(\cos \left(\frac{2 \pi t}{\omega}\right)\right)
$$

with linear, cyclic and exponential parts, which was proposed in [6] and applied in [22]. To generate values from (4), the thinning method (see, e.g., [18]) is then used. Because of using (4), we assume that the premium in (1) is a constant, fixed value for some deterministic moment $T$, and

$$
p(T)=\left(1+\nu_{p}\right) \mathbb{E} C_{i} \int_{0}^{T} \lambda_{\mathrm{NHPP}}(s) d s
$$

where $\nu_{p}$ is a safety loading (security loading) of the insurer (usually about $10 \%-20 \%$, see, e.g, [2]).

In this paper, the classical risk reserve process (1) is generalized to take into account additional financial and insurance instruments (like a catastrophe bond), a penalty function, and the value of money in time (which is modelled using a stochastic interest rate, i.e., the one-factor Vasicek model, given by $\left.d r_{t}=\kappa\left(\theta-r_{t}\right) d t+\sigma d W_{t}\right)$. We enrich the classical insurance portfolio with two additional layers: a catastrophe bond and an external (e.g., governmental or from another institution) help. There are also other possible layers (like a reinsurance contract, see, e.g., [22]).

A catastrophe bond is an example of a complex financial-insurance instrument. When it is issued, the insurer pays an insurance premium $p_{\mathrm{cb}}$ in exchange for coverage, when a triggering point (usually some catastrophic event, like an earthquake) occurs. The investors purchase insurance-linked security for cash. The above-mentioned premium and cash flows are usually managed by an SPV (Special Purpose Vehicle), which also issues the catastrophe bonds. The investors 
hold the issued assets, whose coupons and/or principal depend on the occurrence of the mentioned triggering point. If such a catastrophic event occurs during the specified period, then the SPV compensates the insurer with a payment $f_{\mathrm{cb}}^{i}\left(U_{T}^{*}\right)$ and the cash flows for the investors are changed. Usually, these flows are lowered, i.e. there is full or partial forgiveness of the repayment of principal and/or interest. However, if the triggering point does not occur, the investors usually receive the full payment from a cat bond (see, e.g., $[7,18,20])$.

We assume that the mentioned insurance premium $p_{\mathrm{cb}}$ is proportional to both a part $\alpha_{\mathrm{cb}}$ of the whole price of the single catastrophic bond $I_{\mathrm{cb}}$, and to a number of the issued bonds $n_{\mathrm{cb}}$, i.e. $p_{\mathrm{cb}}=\alpha_{\mathrm{cb}} n_{\mathrm{cb}} I_{\mathrm{cb}}$. To calculate the price $I_{\mathrm{cb}}$ of a catastrophe bond, the martingale method together with the MC simulations are applied (see, e.g., [11,12,18]).

The third layer is an external (e.g., governmental or related to another enterprise from the insurer's consortium) help of the value $f_{\mathrm{hlp}}\left(U_{T}^{*}\right)$. We assume, that this help is supplied only if the losses surpass some given minimal limit $A_{\mathrm{hlp}}$, and it is also limited to some maximal value $B_{\mathrm{hlp}}$. In some way, this help is similar to a reinsurance contract, but, contrary to such an instrument, there is no additional payment for this help and its limits can be modelled in an imprecise way (see Sect.4). Moreover, we assume that only part $\alpha_{\mathrm{hlp}}$ of this help lowers the expenditures of the insurer, because this help can be directed to both the insureds and the insurer. In practice, the formal requirements and the legal regulations can state that such external help is not allowed. But special financial help from the government was directed to banks (which were "too big to fail") during the global financial crisis in 2007-08 and this can happen again under some extreme circumstances. There is also a list, which was published by the Financial Stability Board, of "specially treated" insurance companies (also "too big to fail"). Moreover, it may be profitable even for smaller insurers to analyse the whole set of possible scenarios during their stress tests to check if with and without the external help their portfolios lead to similar levels of probabilities of insolvency.

And in real-life situations, bankruptcy can be very dangerous for the insurer. Apart from simple lack of necessary funds, this event could lead to additional financial problems, bankruptcy of other enterprises, problems with the law, reputational damages, etc. Therefore, we introduce a special penalty function $f_{\text {pen }}\left(R_{T}\right)$ which is related to the bankruptcy event itself or its value (see, e.g., [22]). Then we have a constant penalty function

$$
f_{\text {pen }}\left(R_{T}\right)=b_{\text {pen }} \mathbb{1}\left(R_{T}<0\right)
$$

or, e.g., a linear penalty function (related directly to a level of the bankruptcy)

$$
f_{\text {pen }}\left(R_{T}\right)=a_{\text {pen }} \max \left\{0,-R_{T}\right\}+b_{\text {pen }} \mathbb{1}\left(R_{T}<0\right),
$$

where $a_{\text {pen }}, b_{\text {pen }}$ are respective parameters.

Taking into account the previously assumed value of money in time and the additional layers, the classical risk reserve process (1) should be modified into the form 


$$
\begin{aligned}
R_{T}=\mathrm{FV}_{T}\left(u-p_{\mathrm{cb}}\right)+ & \mathrm{FV}_{T}(p(T))-\mathrm{FV}_{T}\left(C_{T}^{*}\right)+n_{\mathrm{cb}} f_{\mathrm{cb}}^{i}\left(U_{T}^{*}\right) \\
& +\alpha_{\mathrm{hlp}} \mathbb{1}\left(U_{T}^{*} \geq A_{\mathrm{hlp}}\right) f_{\mathrm{hlp}}\left(U_{T}^{*}\right)
\end{aligned}
$$

where $\mathrm{FV}_{T}($.$) is future value of the cash flow in time T$, and together with the penalty function we have

$$
R_{T}^{*}=R_{T}-f_{\text {pen }}\left(R_{T}\right)
$$

In the following, we will be interested in estimation of some important characteristics of the introduced insurer's portfolio, like $\mathbb{E} R_{T}$ (the mean of the generalized risk reserve process in $T$ ), $\operatorname{Pr}\left(R_{T}<0\right.$ ) (probability of the ruin in the finite time horizon $T$ ), $\mathbb{E} R_{T}^{*}$ (the mean of the generalized risk reserve process with the penalty function).

\section{$3 \quad$ Fuzzy Numbers and Shadowed Sets}

In Sect. 4, the general approach to the insurer's portfolio based on both crisp and imprecise settings is discussed. There are many mathematical setups which can be used to model impreciseness. We focus on trapezoidal fuzzy numbers (TPFNs), L-R fuzzy numbers (left-right fuzzy numbers, LRFNs) and shadowed sets (SHSs), but other types of fuzzy sets can be also applied (see, e.g., [1,3, $8,11,13,15,16,18]$ for additional definitions, notation and applications of fuzzy numbers and shadowed sets). In the case of TPFN, its membership function has the form

$$
A(x)= \begin{cases}\frac{x-a_{1}}{a_{2}-a_{1}} & \text { if } a_{1}<x \leqslant a_{2}, \\ 1 & \text { if } a_{2} \leqslant x \leqslant a_{3}, \\ \frac{a_{4}-x}{a_{4}-a_{3}} & \text { if } a_{3} \leqslant x<a_{4}, \\ 0 & \text { otherwise }\end{cases}
$$

where $a_{1}, a_{2}, a_{3}, a_{4} \in \mathbb{R}$, and $a_{1} \leqslant a_{2} \leqslant a_{3} \leqslant a_{4}$. A trapezoidal fuzzy number $\tilde{a}$ will be further denoted as $\left[a_{1}, a_{2}, a_{3}, a_{4}\right]$, and its $\alpha$-level cuts are given by intervals $\tilde{a}[\alpha]=\left[a_{L}[\alpha], a_{U}[\alpha]\right]$. If $a_{2}=a_{3}$, then $\tilde{a}$ is said to be a triangular fuzzy number (TRFN) and we have $\tilde{a}=\left[a_{1}, a_{2}, a_{4}\right]$. The operations on fuzzy numbers are defined as in [3].

An SHS $S$ in a universe of discourse $X$ is a set-valued mapping $S: X \rightarrow$ $\{0,[0,1], 1\}$ interpreted as follows: all elements of $X$ for which $S(x)=1$ are called a core of $S$ and they embrace all elements that are fully compatible with the concepts conveyed by $S$, all elements of $X$ for which $S(x)=0$ are completely excluded from the concept described by $S$, and all elements of $X$ for which $S(x)=[0,1]$, called a shadow, are uncertain. The usage of the unit interval for the shadow shows that any element from this shadow could be excluded or exhibit partial membership or could be fully allocated to $S$. In the following we consider $X=\mathbb{R}$, then a SHS will be denoted by $\left[s_{1}, s_{2}, s_{3}, s_{4}\right]_{\mathrm{SH}}$, where its core is given by the interval $\left[s_{2}, s_{3}\right]$, its shadow by $\left(s_{1}, s_{2}\right) \cup\left(s_{3}, s_{4}\right)$, and its support by $\left[s_{1}, s_{4}\right]$. 
There exist important links between concepts of a fuzzy set and a shadowed set. Based on the initial fuzzy set, a corresponding shadowed set, that captures "the essence" of this fuzzy set, reduces computational efforts related to a membership function (because only two "cuts" are necessary, instead of all possible $\alpha \in[0,1]$ ), and simplifies the interpretation, can be constructed (see, e.g., [13]). This resultant shadowed set is created from the initial fuzzy set using an elevation of some membership values ("close to 1" or "high enough") and with a reduction of others (which are "close to 0" or "low enough"). In [8], the respective procedure, which is related to the optimization of two weighting functions, was introduced.

\section{Imprecise Approaches}

In $[19,20,22]$, the models of the insurer's portfolios with only some parameters given by fuzzy numbers have been described. Now we present a generalization of this approach where all respective parameters are described by imprecise values (if such an assumption is appropriate in the considered real-life case) or crisp (i.e. real) values (when the respective parameters are precisely stated, e.g., in trade agreements). In Sect.5, we focus on TRFNs/TPFNs and SHSs to model the mentioned impreciseness but other types of fuzzy numbers (like LRFNs) can be also applied.

Let us suppose, that our aim is to calculate the value of some function $f(x)$ for $x$, e.g., $\mathbb{E} R_{T}^{*}$ (using its respective estimator, i.e. the average $\bar{R}_{T}^{*}$ based on the MC approach) for $a$. To approximate a fuzzy value $\tilde{f}(\tilde{x})$ for a fuzzy counterpart $\tilde{x}$, monotonicity of $f(x)$ should be checked. If $f(x)$ is a non-decreasing function (or a non-increasing, respectively), then for the given $\alpha \in[0,1]$, the respective $\alpha$-cut of $\tilde{f}(\tilde{x})$ is calculated as the crisp interval $\left[f\left(x_{L}[\alpha]\right), f\left(x_{U}[\alpha]\right)\right]$ (or $\left[f\left(x_{U}[\alpha]\right), f\left(x_{L}[\alpha]\right)\right]$, respectively), following the Zadeh's extension rule (see also, e.g., [21]). If monotonicity of $f(x)$ is more complex or, e.g., unknown, then this interval is given as $\left[\min \left\{f\left(x_{L}[\alpha]\right), f\left(x_{U}[\alpha]\right)\right\}, \max \left\{f\left(x_{L}[\alpha]\right), f\left(x_{U}[\alpha]\right)\right\}\right]$, which complicates the necessary calculations. This step is repeated for all desired values of $\alpha$ (usually we start from $\alpha=0$ with some increment $\Delta \alpha>0$ till $\alpha=1$ ), with an additional approximation of "missing" $\alpha$-level sets based on some (rather simple, e.g., linear) function. In the same manner, probability of the ruin in the finite time $T$ can be estimated using $q_{\text {ruin }}=n_{\text {ruin }} / n$ (i.e. ratio of trajectories with final ruin to number of all trajectories of the portfolio) and its fuzzy counterpart $\tilde{q}_{\text {ruin }}$.

This approach can be generalized, if more than only one parameter $x$ is fuzzified, e.g., if we would like to find $\tilde{R}_{T}^{*}$ (i.e. the fuzzy counterpart for $\bar{R}_{T}^{*}$ ) for both $\tilde{a}$ and $\tilde{b}$ (i.e. the fuzzy counterparts of $a$ and $b$ ). A respective example for $\tilde{R}_{T}^{*}$ and some parameters of the considered models is summarized in Table 1 , where the plus sign indicates non-decreasing dependency and the minus sign non-increasing dependency. In the case of $\tilde{q}_{\text {ruin }}$, these dependencies are reverse. 
Table 1. Examples of dependencies between $\mathbb{E} R_{T}^{*}$ and some parameters of the models.

\begin{tabular}{l|l|l|l|l|l|l|l}
\hline$a$ & $b$ & $\alpha_{\text {claim }}$ & $\zeta_{\text {LN }}$ & $a_{\text {pen }}$ & $b_{\text {pen }}$ & $A_{\text {hlp }}$ & $B_{\text {hlp }}$ \\
\hline- & - & - & - & - & - & - & + \\
\hline
\end{tabular}

The similar approach can be also applied if the parameters are described by SHSs, but then only two "levels" (i.e. $\alpha=0,1$ ) for the respective simulations should be used, and the whole idea is related to interval calculations. The application of SHSs instead of fuzzy numbers significantly reduces the necessary time of the MC simulations, but it can also lead to different results (see Sect. 5). This approach can be profitable if monotonicity of the considered function for some parameters is too complex to asses analytically.

\section{$5 \quad$ Numerical Simulations}

To make our simulations more realistic, we focus on the values of the parameters, which have been fitted to real-life data in the literature. But because many future aspects of the considered models are uncertain, some of these parameters are transformed to imprecise values, i.e. they are given as fuzzy numbers (modelled by TPFNs/TRFNs) or SHSs. Then, to describe each $U_{i}$, we apply the lognormal distribution with the parameters $\zeta_{\mathrm{LN}}, \sigma_{\mathrm{LN}}$ fitted in [6] to real-life data (collected by PCS, USA). The parameters of the intensity function (4) have been also fitted in [6]. The parameters of the one-factor Vasicek model are based on estimators from [4]. To describe the limits for a catastrophe bond and external help, quantiles of the cumulated value of the losses for the process (3), denoted by $Q_{C_{T}^{*}}(x)$, are also applied (see also, e.g., [20]). A piecewise function is used as the payment function $f_{\mathrm{cb}}^{i}\left(U_{T}^{*}\right)$ of the issued catastrophe bond (for additional details about these payments and the pricing method, see [18,22]).

Some of the above-mentioned parameters are given as imprecise values in the following simulations. To make our analysis clearer, we limit the number of these parameters, however, the approach presented in Sect. 4 is more general.

The above-mentioned impreciseness can have many sources. It can be related to future unknown behaviour of the introduced models (like the parameters in (4) or for the distribution of $U_{i}$, so the fuzzified value $\tilde{a}$ reflects our doubts concerning the future possible increase of intensity of the number of the catastrophic events caused by the global warming effect), lack of our knowledge because of the rarity of similar events in the past (then the experts' knowledge has to be applied, e.g., to estimate the parameters of the penalty function or the external help from another enterprise, so $\tilde{A}_{\mathrm{hlp}}$ is related to the unknown minimum level of the possible external help, which can be smaller or bigger depending on political restrictions or available financial resources of another company), rapid changes in the market or lack of full information concerning the given region or the specified segment of the market (e.g., if there is a new competitor on the market 
or a natural catastrophe causes the losses only in some region or to some kind of properties, hence the share of the insurer in this market can be imprecise). Other parameters can be given exactly as crisp values because, e.g., they are precisely stated in contracts or directly known (like $p_{\mathrm{cb}}, u$ or the parameters of a cat bond). We consider one-year time horizon in our simulations (i.e. $T=1$ ), and for each example one million simulations are used.

First, we assume that the imprecise values are modelled by TPFNs/TRFNs, so we set

$$
\begin{gathered}
u=Q_{C_{T}^{*}}(0.25), \nu_{p}=0.1, I_{\mathrm{cb}}=0.809896, \alpha_{\mathrm{cb}}=0.1, n_{\mathrm{cb}}=1000, w_{1}=1, \\
K_{0}=Q_{C_{T}^{*}}(0.7), K_{1}=Q_{C_{T}^{*}}(0.9), \alpha_{\mathrm{hlp}}=0.5, p_{\mathrm{hlp}}=1, \\
\tilde{A}_{\mathrm{hlp}}=\left[Q_{C_{T}^{*}}(0.95)-1000, Q_{C_{T}^{*}}(0.95), Q_{C_{T}^{*}}(0.95)+1000\right], \\
\tilde{B}_{\mathrm{hlp}}=\left[Q_{C_{T}^{*}}(0.99)-1000, Q_{C_{T}^{*}}(0.99), Q_{C_{T}^{*}}(0.99)+1000\right], \\
\kappa=0.1779, \theta=0.086565, r_{0}=0.03, \sigma=0.02, a=[23.93,24.93,25.93], \\
b=[0.025,0.026,0.027], c=5.6, d=7.07, m=10.3, \omega=4.76, \\
\zeta_{\mathrm{LN}}=[18.08,18.58,19.08], \sigma_{\mathrm{LN}}=1.49, \alpha_{\mathrm{claim}}=[0.4,0.5,0.6], \\
a_{\text {pen }}=[1,2,3,4], b_{\mathrm{pen}}=[5,10,15,20],
\end{gathered}
$$

then, e.g., the insurer issued 1000 catastrophe bonds, the parameter $a$ in the intensity function (4) is "about its value estimated in [6] plus/minus 1", deterministic share of the insurer in the insurance market $\alpha_{\text {claim }}$ is "about $50 \%$ plus $/$ minus $10 \%$, the constant value $b_{\text {pen }}$ in the penalty function is given by TPFN with the core $[10,15]$ and the support $[5,20]$, etc.

During our simulations, the insurer's portfolios without the penalty function and without the external help (Example F1), without the penalty function and with the external help (Example F2), and their counterparts with the linear penalty function (Example F3/Example F4), have been analysed. The simulated values of $\tilde{R}_{T}^{*}$ and $\tilde{q}_{\text {ruin }}$ can be found in Fig. 1 and Fig. 2, respectively. They are clearly LRFNs with rather wide supports (especially in the cases without the external help), very narrow cores, left-skewed (in the case of $\tilde{R}_{T}^{*}$ ) or right-skewed ones $\left(\tilde{q}_{\text {ruin }}\right)$. Interestingly, the existence of the external help or/and the linear penalty function has limited influence on the right arms of $\tilde{R}_{T}^{*}$ and the left arms of $\tilde{q}_{\text {ruin }}$ (whereas very significant on their left/right counterparts, respectively). The external help narrows the supports of both $\tilde{R}_{T}^{*}$ and $\tilde{q}_{\text {ruin }}$ almost twice, but its influence on the cores of $\tilde{R}_{T}^{*}$ is more limited.

The insurer can easily evaluate the impacts of different scenarios on his financial results using the simulated outputs. For example, the insurer can be interested if the increasing intensity of the number of natural catastrophes in future can lead to additional problems with his portfolio. Then, instead of the values of $a, b$ given in (11), TPFNs with wider right arms (because we suspect that the intensity will be rather bigger than smaller)

$$
a=[23.93,24.93,26.93,28.93], b=[0.025,0.03,0.026,0.028]
$$




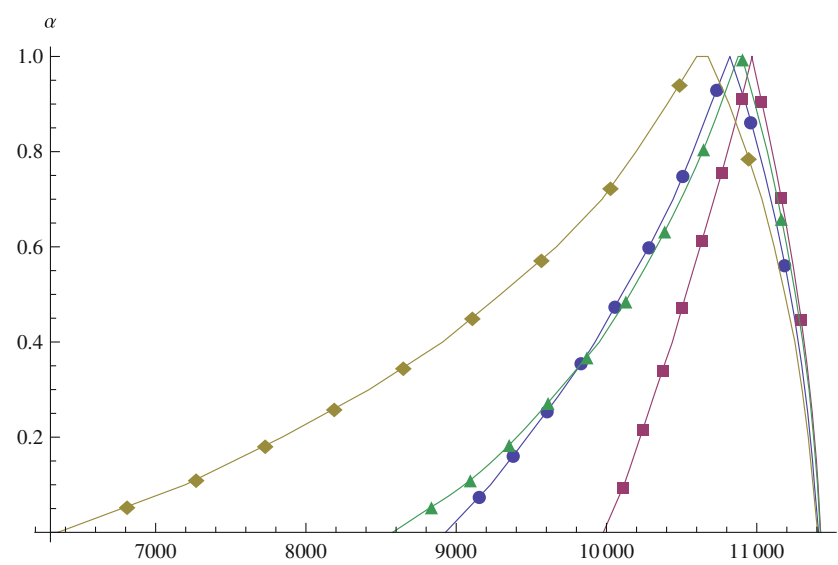

Fig. 1. Simulated values of $\tilde{R}_{T}^{*}$ in Example F1 (circles), Example F2 (rectangles), Example F3 (diamonds), Example F4 (triangles).



Fig. 2. Simulated values of $\tilde{q}_{\text {ruin }}$ in Example F1/F3 (circles), Example F2/F4 (rectangles).

can be used, together with the setting without the external help and the penalty function (Example F5). The simulated value of $\tilde{R}_{T}^{*}$ is similar to the one for Example F1 (see Fig. 3a), but the core in Example F5 is wider. Hence, the obtained results for $\alpha=1$ are given by the wider interval. On the other hand, the insurer can suspect that his share in the insurance market is given by more imprecise TPFN

$$
\alpha_{\text {claim }}=[0.3,0.4,0.6,0.7]
$$


than the value set in (11) (Example F6). In this case, both the core and the support of $\tilde{R}_{T}^{*}$ are much wider than in Example F1 (see Fig. 3b), so the implicated impreciseness of this result is really high.

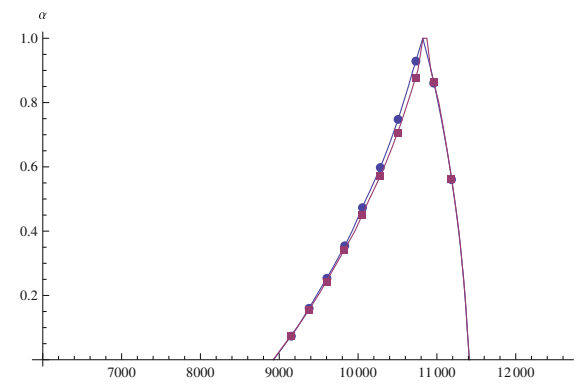

(a) Example F1 (circles) vs F5 (rectangles)

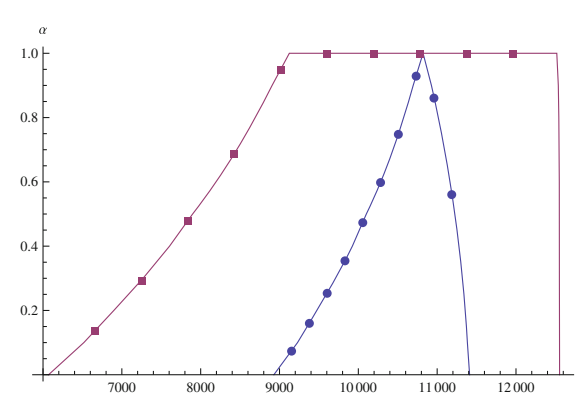

(b) Example F1 (circles) vs F6 (rectangles)

Fig. 3. Simulated values of $\tilde{R}_{T}^{*}$.

Other important characteristics of the insurer's portfolio can be also found similarly. In Table 2 some examples of statistics for the final value of the portfolio in Example $\mathrm{F} 1$ for $T=1$ and $\alpha=0,1$ are given. Especially the median can be interesting for the insurer, because of the skewness which is seen for $\tilde{R}_{T}^{*}$.

Table 2. Selected statistics of the portfolio in Example F1.

\begin{tabular}{l|l|l|l|l|l}
\hline & Minimum & $Q_{1}$ & Median & $Q_{3}$ & Maximum \\
\hline$\alpha=0$ & {$[-260405,-85962]$} & {$[6450,10657]$} & {$[10074,11729]$} & {$[12561,13285]$} & {$[15537,23950]$} \\
\hline$\alpha=1$ & {$[-227392,-222036]$} & {$[9224,9225]$} & {$[11443,11448]$} & {$[13146,13148]$} & {$[18407,18802]$} \\
\hline
\end{tabular}

Now we compare the simulated outputs when, instead of fuzzy numbers, their SHSs counterparts are applied. To do this, we apply the procedure from [8] to transform the fuzzy numbers in (11) to respective SHSs. We consider similar examples to the previous ones, i.e. without the penalty function and without the external help (Example S1 instead of F1), without the penalty function and with the external help (S2 instead of F1), etc. Then, the simulated values of $\tilde{R}_{T}^{*}$ and $\tilde{q}_{\text {ruin }}$ can be found in Fig. 4 and Fig. 5, respectively. The previously obtained plots for Examples F1-F4 are also added there for easier comparison of both approaches.

In general, the obtained SHSs are "similar" to the previously considered LRFNs, especially their skewness can be directly seen. However, the supports of SHSs are much shorter and their cores wider. Therefore, instead of LRFNs with almost the "crisp" cores (like in Example F1 or F2), we obtain rather wide 


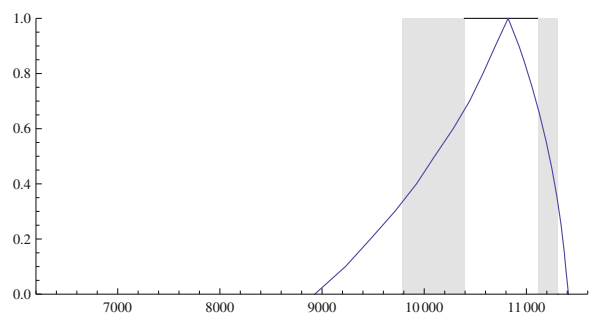

(a) Example S1 vs F1

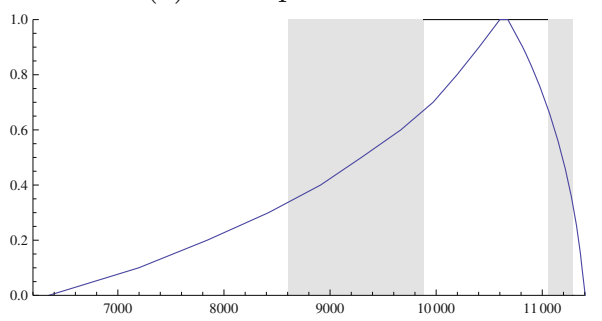

(c) Example S3 vs F3

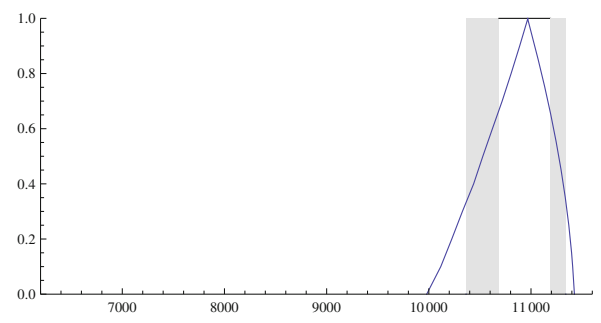

(b) Example S2 vs F2

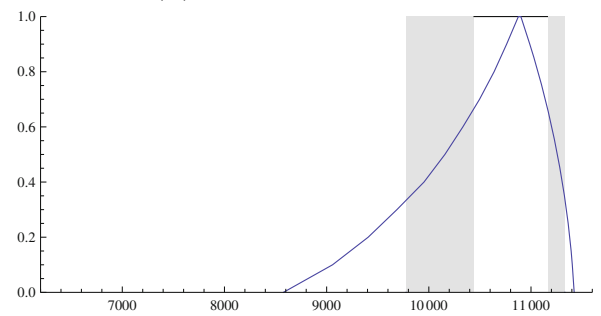

(d) Example S4 vs F4

Fig. 4. Simulated values of $\tilde{R}_{T}^{*}$.

intervals for "fully compatible" values. And, on the contrary, the shorter supports can also lead us to different (from the insurer's point of view) evaluation of "fully excluded" values. It can be treated as some drawback of the approach based on SHSs, while its advantage is related to the previously mentioned reduction of time of simulations. However, the general shapes of the calculated SHSs and LRFNs are very similar, so the insurer can obtain a general idea concerning impreciseness of the evaluated values even for the approach based on SHSs. This kind of comparison between both approaches can be also made for other areas of applied sciences (see, e.g., [17]).

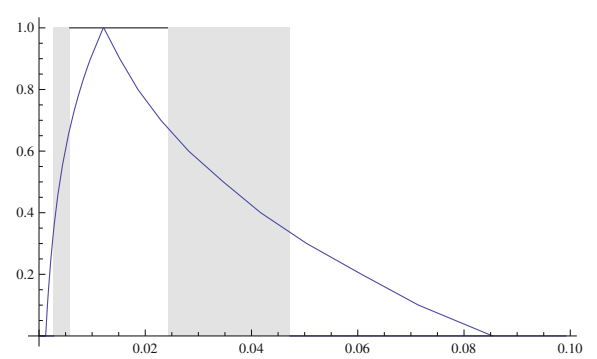

(a) Example S1/S3 vs F1/F3

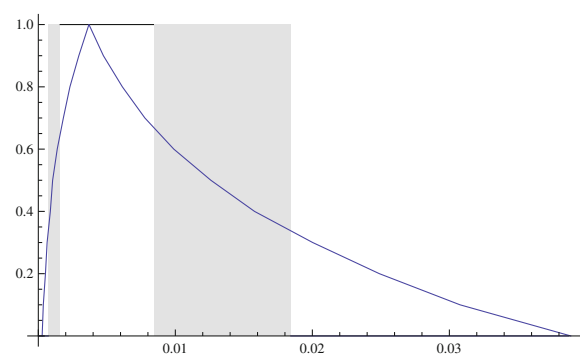

(b) Example S2/S4 vs F2/F4

Fig. 5. Simulated values of $\tilde{q}_{\text {ruin }}$. 
Table 3. Selected statistics of the portfolio in Example S1.

\begin{tabular}{l|l|l|l|l|l}
\hline & Minimum & $Q_{1}$ & Median & $Q_{3}$ & Maximum \\
\hline$\alpha=0$ & {$[-218936,-113416]$} & {$[7730,10349]$} & {$[10632,11722]$} & {$[12780,13332]$} & {$[16464,23950]$} \\
\hline$\alpha=1$ & {$[-383767,-149285]$} & {$[8319,9881]$} & {$[11124,11628]$} & {$[12970,13277]$} & {$[17638,18802]$} \\
\hline
\end{tabular}

Other statistics of the final value of the portfolio can be also compared as in the case of Table 2 for Example F1 and Table 3 for its SHS counterpart (i.e. Example S1). To provide additional insight into statistical characteristics of the above-mentioned values, respective quantile plots are plotted for the left and right ends of the cores for both of these examples in Fig. 6. The obtained results are very similar for "middle quantiles" (like 0.5 th quantile) and differ significantly for lower and upper quantiles, but the overall shapes of the plots are comparable.

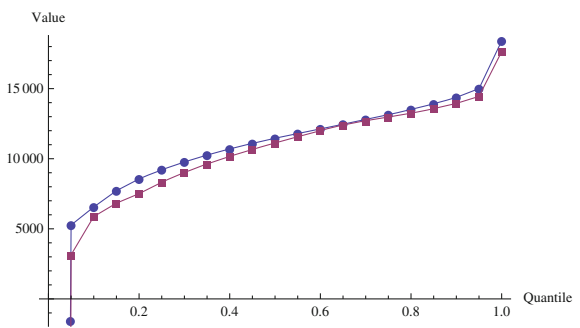

(a) Left end of the core

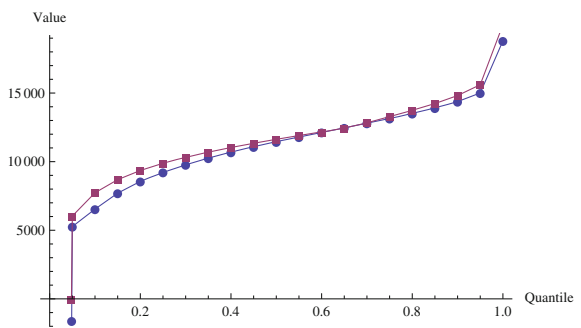

(b) Right end of the core

Fig. 6. Quantile plots for Example F1 (circles) vs S1 (rectangles).

\section{Conclusion}

In this paper, the general imprecise approach to the model of the risk reserve process is discussed. The presented insurance portfolio consists of the classical risk reserve process, the issued catastrophe bond and the external help. In the case of the insurer's bankruptcy, the special penalty function is applied. Apart from the general approach, two special cases, when the considered parameters are given by triangular/trapezoidal fuzzy numbers or shadowed sets, are discussed in a more detailed way. Then these cases are applied and compared using the Monte Carlo simulations for which the parameters based on real-life values are used. The obtained results for both of the previously mentioned approaches seem to be similar, but there are some important differences. The calculated fuzzy numbers give us more detailed information, especially in the case of their cores (i.e. about "sure values"), which can be very useful for the insurer (because of the above-mentioned "certainty" of these values for $\alpha=1$ ). Exact knowledge of the whole membership function of fuzzy number has also a serious drawback related to the expenditure of time of necessary simulations (let's say, 5-10 times 
more than in the case of the second approach). In the contrary, an application of shadowed sets leads us to a fast, intuitively understandable but rather coarse approximation of the expected output. It can be useful for the insurer as a first attempt to solve the considered problem. Nevertheless, the overall "shapes" of the outputs for both approaches remain very similar.

\section{References}

1. Ángeles Gil, M., Hryniewicz, O.: Statistics with imprecise data. In: Meyers, R.A. (ed.) Computational Complexity: Theory, Techniques, and Applications, pp. 30523063. Springer, New York (2012). https://doi.org/10.1007/978-1-4614-1800-9

2. Asmussen, S., Albrecher, H.: Ruin Probabilities, 2nd edn. World Scientific, Singapore (2010)

3. Ban, A., Coroianu, L., Grzegorzewski, P.: Fuzzy Numbers: Approximations, Ranking and Applications. Polish Academy of Sciences, Warsaw (2015)

4. Chan, K.C., Karolyi, G.A., Longstaff, F.A., Sanders, A.B.: An empirical comparison of alternative models of the short-term interest rate. J. Finance 47(3), 1209-1227 (1992)

5. Ghasemalipour, S., Fathi-Vajargah, B.: The mean chance of ultimate ruin time in random fuzzy insurance risk model. Soft Comput. 22(12), 4123-4131 (2017). https://doi.org/10.1007/s00500-017-2629-0

6. Giuricich, M.N., Burnecki, K.: Modelling of left-truncated heavy-tailed data with application to catastrophe bond pricing. Physica A 525, 498-513 (2019)

7. Goda, K.: Seismic risk management of insurance portfolio using catastrophe bonds. Comput.-Aided Civ. Inf. Eng. 30(7), 570-582 (2015)

8. Grzegorzewski, P.: Fuzzy number approximation via shadowed sets. Inf. Sci. 225, 35-46 (2013)

9. Kulczycki, P., Charytanowicz, M., Dawidowicz, A.L.: A convenient ready-to-use algorithm for a conditional quantile estimator. Appl. Math. Inf. Sci. 9(2), 841-850 (2015)

10. Ma, J., Sun, X.: Ruin probabilities for insurance models involving investments. Scand. Actuar. J. 2003(3), 217-237 (2003)

11. Nowak, P., Romaniuk, M.: Catastrophe bond pricing for the two-factor Vasicek interest rate model with automatized fuzzy decision making. Soft Comput. 21(10), 2575-2597 (2015). https://doi.org/10.1007/s00500-015-1957-1

12. Nowak, P., Romaniuk, M.: Valuing catastrophe bonds involving correlation and CIR interest rate model. Comput. Appl. Math. 37(1), 365-394 (2016). https:// doi.org/10.1007/s40314-016-0348-2

13. Pedrycz, W.: Shadowed sets: representing and processing fuzzy sets. IEEE Trans. Syst. Man Cybern. Part B (Cybern.) 28(1), 103-109 (1998)

14. Pizzutilo, F., Venezia, E.: Are catastrophe bonds effective financial instruments in the transport and infrastructure industries? Evidence from international financial markets. Bus. Econ. Horiz. 14(2), 256-267 (2018)

15. Romaniuk, M.: On simulation of maintenance costs for water distribution system with fuzzy parameters. Eksploat. Niezawodn. - Maint. Reliab. 18(4), 514-527 (2016)

16. Romaniuk, M.: Optimization of maintenance costs of a pipeline for a V-shaped hazard rate of malfunction intensities. Eksploat. Niezawodn. - Maint. Reliab. 20(1), 46-56 (2018) 
17. Romaniuk, M.: Estimation of maintenance costs of a pipeline for a U-shaped hazard rate function in the imprecise setting. Eksploat. Niezawodn. - Maint. Reliab. 22(2), 352-362 (2020)

18. Romaniuk, M., Nowak, P.: Monte Carlo Methods: Theory, Algorithms and Applications to Selected Financial Problems. Institute of Computer Science Polish Academy of Sciences, Warsaw (2015)

19. Romaniuk, M.: Analysis of the insurance portfolio with an embedded catastrophe bond in a case of uncertain parameter of the insurer's share. In: Wilimowska, Z., Borzemski, L., Grzech, A., Świątek, J. (eds.) Information Systems Architecture and Technology: Proceedings of 37th International Conference on Information Systems Architecture and Technology - ISAT 2016 - Part IV. AISC, vol. 524, pp. 33-43. Springer, Cham (2017). https://doi.org/10.1007/978-3-319-46592-0_3

20. Romaniuk, M.: Insurance portfolio containing a catastrophe bond and an external help with imprecise level - a numerical analysis. In: Kacprzyk, J., Szmidt, E., Zadrożny, S., Atanassov, K.T., Krawczak, M. (eds.) IWIFSGN/EUSFLAT-2017. AISC, vol. 643, pp. 256-267. Springer, Cham (2018). https://doi.org/10.1007/9783-319-66827-7 23

21. Romaniuk, M.: On some applications of simulations in estimation of maintenance costs and in statistical tests for fuzzy settings. In: Steland, A., Rafajłowicz, E., Okhrin, O. (eds.) SMSA 2019. SPMS, vol. 294, pp. 437-448. Springer, Cham (2019). https://doi.org/10.1007/978-3-030-28665-1_33

22. Romaniuk, M.: Simulation-based analysis of penalty function for insurance portfolio with embedded catastrophe bond in crisp and imprecise setups. In: Wilimowska, Z., Borzemski, L., Świątek, J. (eds.) ISAT 2018. AISC, vol. 854, pp. 111-121. Springer, Cham (2019). https://doi.org/10.1007/978-3-319-99993-7_11

23. Yan, C., Liu, Q., Liu, J., Liu, W., Li, M., Qi, M.: Payments per claim model of outstanding claims reserve based on fuzzy linear regression. Int. J. Fuzzy Syst. 21, 1950-1960 (2019). https://doi.org/10.1007/s40815-019-00617-x 